\title{
Steady State and (Bi-) Stability Evaluation of Simple Protease Signalling Networks
}

\author{
Thomas Eißing a,*, Steffen Waldherr ${ }^{\text {a }}$, Frank Allgöwer ${ }^{a}$, \\ Peter Scheurich ${ }^{\mathrm{c}}$, and Eric Bullinger ${ }^{\mathrm{a}, \mathrm{b}}$ \\ ${ }^{a}$ Institute for Systems Theory and Automatic Control, University of Stuttgart, \\ 70550 Stuttgart, Germany. \\ ${ }^{\mathrm{b}}$ The Hamilton Institute, National University of Ireland, Maynooth, Co. Kildare, \\ Ireland. \\ ${ }^{\mathrm{c}}$ Institute of Cell Biology and Immunology, University of Stuttgart, 70569 \\ Stuttgart, Germany.
}

\begin{abstract}
Signal transduction networks are complex, as are their mathematical models. Gaining a deeper understanding requires a system analysis. Important aspects are the number, location and stability of steady states. In particular, bistability has been recognised as an important feature to achieve molecular switching. This paper compares different model structures and analysis methods particularly useful for bistability analysis.

The biological applications include proteolytic cascades as, for example, encountered in the apoptotic signalling pathway or in the blood clotting system. We compare three model structures containing zero-order, inhibitor and cooperative ultrasensitive reactions, all known to achieve bistability. The combination of phase plane and bifurcation analysis provides an illustrative and comprehensive understanding of how bistability can be achieved and indicates how robust this behaviour is.

Experimentally, some so-called "inactive" components were shown to have a residual activity. This has been mostly ignored in mathematical models. Our analysis reveals that bistability is only mildly affected in the case of zero-order or inhibitor ultrasensitivity. However, the case where bistability is achieved by cooperative ultrasensitivity is severely affected by this perturbation.
\end{abstract}

Key words:

bistability, switch, threshold, signalling, proteases, caspases, apoptosis

\footnotetext{
* Corresponding author: phone: +49 711685 67750, fax: +49 711685 67735, email: eissing@ist.uni-stuttgart.de
} 


\section{Introduction}

Mathematical models of biological signalling processes have been proven useful for more than half a century, e.g. Hodgkin and Huxley (1952), and the interest in pursuing model aided approaches is growing (Ventura et al., 2006). Modelling has been extensively used to uncover principal roles and functions of reaction networks and network elements, e.g. Tyson et al. (2003); Mangan and Alon (2003); Yeger-Lotem et al. (2004); Wolf and Arkin (2003). Important characteristics of mathematical models and the processes described by these models are attraction phenomena such as steady state behaviour or limit cycles (oscillations). Despite the inherent nonlinearity of biological systems, chaotic attractors appear to be of less importance (Shmulevich et al., 2005). Biological processes rather operate in an ordered regime and are robust against perturbations as is now also supported by the analysis of various mathematical models (Stelling et al., 2004).

This article focuses on systems where spatial and stochastic effects can be neglected. These systems can be mathematically represented by ordinary differential equations (ODEs). Qualitative analyses are important to reveal principal system characteristics. Basic qualitative properties of signal transduction models are steady states, limit cycles and transients. System structure and parameter values dictate these properties. Thereby, robustness with respect to parameter changes is important to account for variations between cells or over time.

Bistability is a nonlinear phenomenon relevant in numerous signal transduction systems and allows to interpret many phenomena (Eißing et al., 2004; Ferrell and Xiong, 2001; Ferrell, 1996; Tyson et al., 2003; Thron, 1997; Cherry and Adler, 2000; Qu et al., 2003). The best studied biological example with respect to bistability are kinase cascades as exemplified by the mitogen activated protein kinase (MAPK) cascade (Ferrell and Xiong, 2001; Ferrell, 1997, 1996; Huang and Ferrell, 1996; Markevich et al., 2004; Bhalla et al., 2002). It has been established that bistability generally requires two ingredients (Ferrell and Xiong, 2001; Angeli et al., 2004). The first is an ultrasensitive reaction mechanism. Ultrasensitivity refers to a system response that is more sensitive to changes in the component concentrations than is the normal hyperbolic response given by the Michaelis-Menten equation (Koshland, 1998), e.g. a Hill type response (Weiss, 1997). The second is positive feedback, which can be implemented by only positive (activatory) feedback or an even number of negative (inhibitory) interactions along the loop (Angeli et al., 2004). However, both ingredients are only necessary but not sufficient to generate bistability. Whether the system is then bistable or not is strongly dependent on the parameter values (Ferrell and Machleder, 1998; Xiong and Ferrell, 2003)(see also Section 3). Despite several case studies, the development of general approaches 
to determine bistability is rather difficult. Recently, promising approaches for special system classes have been described (Angeli et al., 2004; Angeli, 2006). Admittedly, due to the restriction to special classes the widespread use of these approaches is still limited so far.

Phase-plane and bifurcation analyses enable an illustrative insight into the qualitative system behaviour (Strogatz, 2001). They allow an efficient analysis of steady states with respect to number, location and stability. Classical phase-plane analysis is considering the phase-space of second order systems. It is able to illustrate the vector field governing the differential equations, or selected properties thereof, for a fixed parameter set. If larger systems can be reduced by the elimination of variables, they are also amenable to a phaseplane analysis, e.g. Tyson et al. (2003). Bifurcation analyses explore how phase space properties like steady states and limit cycles depend on the parameters. Restrictions are only introduced by numerical accuracy, computational power and graphical illustration.

In this study, we will explore and compare different mechanisms for generating a bistable behaviour. Systematic comparisons of these mechanisms have so far been scarce and in addition we chose a less well studied class of biological reactions, namely systems of proteins that can mutually cleave and thereby activate each other. This kind of reaction is frequently encountered in biology, e.g. during programmed cell death or blood clotting. Especially the irreversibility of the involved modification, i.e. protein cleavage, renders the problem special in comparison to other biological reaction systems. For example, in the above mentioned kinase cascade the modification can be easily reversed through a back reaction, i.e. phosphatases catalysing the dephosphorylation.

In Section 2 we will introduce and motivate the models analysed in the following. Section 3 provides an overview on the concept of phase plane analysis at the example of a cooperative model. Section 4 compares three basic reaction mechanisms allowing for a bistable behaviour, i.e. cooperativity, inhibitor binding and back reaction saturation and discusses briefly additional mechanisms. A different viewpoint on the system behaviour in dependence on parameters and possible external inputs is provided in Section 5 through bifurcation analyses at the example of the inhibitor model. In Section 6 we extend these comparisons to include the influence of residual activities of so-called "inactive" components. Although experimentally established, for simplicity this influence has so far been mostly ignored in mathematical models. 


\section{Mathematical models and their biological relevance}

As introduced, we will focus on simple reaction networks describing proteases, i.e. proteins that can cleave other proteins. Several findings as well as the methodological approach are however also applicable to many reaction schemes found in signal transduction.

Section 2.1 provides a short overview of the biological context and relevance of the studied reaction systems that are detailed in Section 2.2.

\subsection{Biological relevance of protease cascades}

Proteases are proteins able to cleave other proteins. They are a wide-spread class of enzymes implicated in diverse metabolic and regulatory functions. This study focuses on protease cascades, i.e. inter-winded protease reactions. A prominent example where this reaction setup can be found is apoptosis.

Apoptosis is a form of programmed cell death which can be executed in every cell. The programme is essential to remove cells that are old, infected or potentially dangerous. Misregulation is implicated in severe pathological alterations (Danial and Korsmeyer, 2004; Hengartner, 2000; Leist and Jaattela, 2001). Caspases, a family of aspartate directed cysteine proteases, are at the heart of apoptosis. Caspases are produced in an inactive pro-form also called zymogen which is activated by proteolytic cleavage. Initiator caspases sense the internal or external status of the cell and once activated, they can cleave and thereby activate downstream executioner caspases. Various feed-back and -forward loops have been described to yield a complex reaction network at the caspase level already (Sohn et al., 2005; Stennicke and Salvesen, 1999; Slee et al., 1999; Van de Craen et al., 1999; Hengartner, 2000).

However, protease cascades are by far not restricted to apoptosis. The blood clotting system heavily relies on the proteolytic cleavage of key regulators and clotting substrates with several inhibitors and feedback loops being known (Dahlbäck, 2000; Zarnitsina et al., 2001; Beltrami and Jesty, 1995). Further, proteases such as cathepsin or metallo-proteases are implicated in many regulatory processes within and immediately outside of the cell, also critically involved in diverse and severe diseases (Overall and López-Otín, 2002; Dash et al., 2003; Grabowskal et al., 2005; Thurmond et al., 2005; Sloane et al., 2005; Yamashima, 2004).

The above overview shows that reaction networks of interacting and mutually activating proteases are frequently used in cellular systems and that one important role is the finding or execution of cellular decisions. Decisions of- 
ten involve molecular switches that convert continuous inputs into discrete outputs to safeguard transitions between different cellular states. When using ODEs to describe these processes, steady states correspond to these cellular states and a switch is commonly represented by a bistable system.

\subsection{Model overview and notation}

We consider the models outlined in Fig. 1. In the simplest form, we consider two proteases $X$ and $Y$ (Fig.1, white background). The inactive protease $X_{i}$ is activated by an input (or external stimulus) $U$ yielding the activated form of the protease $X_{a}$ (reaction rate $r_{0}$ ). $X_{a}$ itself is then able to activate the inactive protease $Y_{i}$ to give $Y_{a}\left(r_{2}\right)$, which can feed-back on $X\left(r_{1}\right)$. In general we use mass action kinetics. We also consider the effects of a cooperative mechanism in the activation of $Y\left(r_{2}\right)$ and compare the results to effects conferred by inhibitors of activated proteases $\left(I_{i}\right.$ and $I_{e}, r_{3}$ and $\left.r_{4}\right)$ or a saturation in the degradation (see below). In apoptosis signalling, $X, Y$ and $I$ correspond to initiator and executioner caspases and inhibitor of apoptosis proteins (IAPs), respectively. Thereby initiator caspases as a first approximation can be viewed as the receptor associated initiator caspases 8 and 10 or the intrinsic pathway initiator caspase 9 . We further study the influence of zymogen activity ( $r_{13}$ and $r_{14}$ omitted in Fig. 1 for clarity), i.e. the inactive proteases have a residual activity and can thus catalyse the same reactions as the activated protease, but with a lower efficiency.

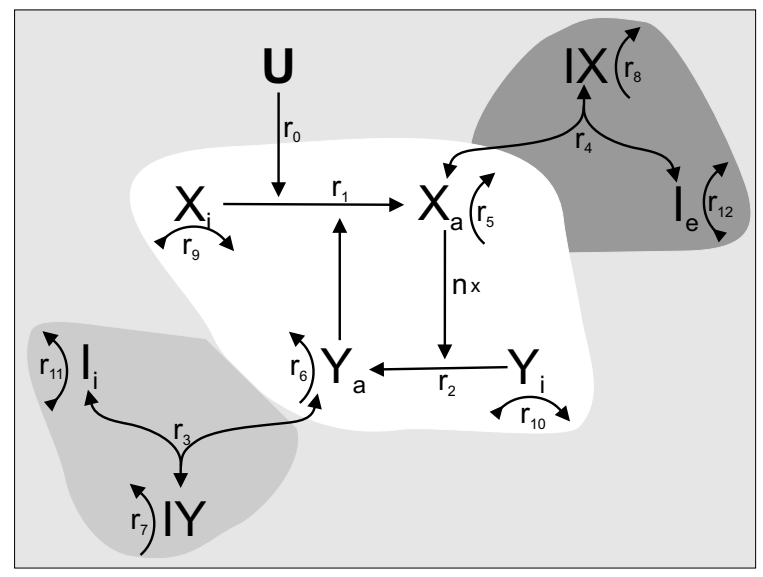

Figure 1. Model outline. Schematic sketch of the models used in this study. Please see Section 2.2 for details.

We will consider a turnover of all involved components $\left(r_{5}-r_{12}\right)$. For this turnover we assume that all components are degraded (with a kinetic proportional to their concentration unless a saturation is assumed), while only the non-activated and non-bound proteins will also be produced (with a kinetic independent of the concentration). Although the biological mechanisms are, 
of course, totally different for producing and degrading pathways, we combine them into one apparent net rate for convenience (turnover rates with arrowheads on both ends).

A turnover is essential because we would like to evaluate the steady state behaviour with a focus on bistability. As we consider proteolytic reactions, it can be assumed that these are essentially irreversible. Thus, negligence of protein turnover would inevitably lead to a complete activation of the proteases in the setup described above once a single molecule would be activated, e.g. by a minor input $U$.

Irreversibility, turnover and inhibitors are essential differences to well studied examples, e.g. the MAPK cascade. These differences also prevent the straightforward application of new powerful methods developed in the framework of monotone systems (Angeli et al., 2004; Angeli, 2006). Although simplifications (as illustrated below) can render some of the models introduced here monotone, we will rely on classical phase plane analysis (which is related but much wider known).

If not indicated otherwise, we do not explicitly consider the input $U$ but rather evaluate the behaviour of the system with $U \equiv 0$. If we consider $U$ as an impulse, we account for the molecules activated by $U$ in the initial conditions of the system without input. Thus, we only explicitly have to account for $U$ when considering a different kind of input signal (e.g. a constant stimulus).

We denote the kinetic rate constants by $k_{j r}$, whereby the index $j$ describes the class the parameter is belonging to, i.e. $k_{c r}$ for catalytic constants involved in the cleavage reactions, $k_{f r}$ and $k_{b r}$ for the forward and backward reaction constants of binding reactions, $k_{d r}$ and $k_{p r}$ for degradation and production terms and $k_{z r}$ for zymogen mediated cleavage reactions. The index $r$ corresponds to the reaction rate number as given in Fig. 1. At the point where we consider saturation effects, we use Michaelis-Menten instead of mass action kinetics and denote the Michaelis-Menten constant with $K_{M r}$ and the maximal rate constant with $k_{m r}$.

Parameter values are explicitly only used in the normalized models introduced in Section 3.2. Due to this normalization, Michaelis-Menten constants correspond to a normalized concentration, $n$ is dimensionless, and all other parameters are in one per unit of time. If not specified otherwise we use the following parameter values $p=\left(k_{c r}, n, k_{f r}, k_{b r}, k_{d r}, k_{p r}, k_{m r}, K_{M r}\right)=(0.01,2.5,1,0.001$, $0.003,0.003,0.003,0.01)$. 


\section{Steady states and their stability in a cooperative model}

As outlined in Section 2.2, we start with a simple model only considering two mutually activating proteases that exist in an active and inactive form. We start with the general description of a model considering a cooperative action of $n$ molecules $X_{a}$ to activate $Y\left(r_{2}\right.$, Fig. 1). A simpler model without cooperativity can be obtained by setting $n=1$. We introduce the external input $U$, but neglect it in the following most of the time. Using the introduced mathematical approach, we obtain the following system of coupled nonlinear ODEs

$$
\begin{aligned}
\dot{X}_{a} & =k_{c 1} \cdot X_{i} \cdot Y_{a}-k_{d 5} \cdot X_{a}+U \cdot X_{i}, \\
\dot{Y}_{a} & =k_{c 2} \cdot Y_{i} \cdot X_{a}^{n}-k_{d 6} \cdot Y_{a} \\
\dot{X}_{i} & =-k_{c 1} \cdot X_{i} \cdot Y_{a}-k_{d 9} \cdot X_{i}+k_{p 9}, \\
\dot{Y}_{i} & =-k_{c 2} \cdot Y_{i} \cdot X_{a}^{n}-k_{d 10} \cdot Y_{i}+k_{p 10} .
\end{aligned}
$$

Introducing the vector $x=\left(X_{a}, Y_{a}, X_{i}, Y_{i}\right)$ the ODE system can be written in the compact form $\dot{x}=f(x)+g(x) \cdot U$.

In Section 3.1 we show an approach to visualize the steady states of the full system in the phase plane. In Section 3.2 we introduce simplifications for clarity not affecting the qualitative behaviour of the system, before providing a phase plane analysis in Section 3.3.

\subsection{Steady state analysis of the full system}

The above ODE system (1)-(4) is nonlinear. The coupling of the ODEs makes the determination of steady states non-trivial. We assume $U \equiv 0$. To obtain the steady states we can partly solve the equation system at steady state and visualize the solution in the phase plane. To eliminate two equations we can solve $f_{3}(x)=0$ for $X_{i}$ and substitute the solution into $f_{1}$ and similarly solve $f_{4}(x)=0$ for $Y_{i}$ and substitute the solution into $f_{2}$. The two equations left provide nullclines in the phase plane whose intersections are steady states

$$
\begin{aligned}
X_{a} & =\frac{k_{c 1} \cdot k_{p 9} \cdot Y_{a}}{k_{d 5} \cdot\left(k_{d 9}+k_{c 1} \cdot Y_{a}\right)}, \\
Y_{a} & =\frac{k_{c 2} \cdot k_{p 10} \cdot X_{a}^{n}}{k_{d 6} \cdot\left(k_{d 10}+k_{c 2} \cdot X_{a}^{n}\right)} .
\end{aligned}
$$

In the general case, this procedure is possible if the equation to be eliminated can be solved for the variable to be eliminated. 


\subsection{Simplifications and normalisation}

The above equations contain several parameters. As shown, deriving a general solution is still straightforward. However, we will now introduce a simplified system and afterwards show the similarities to the full system.

Assuming equal degradation rates for active and inactive proteases, i.e. $k_{d 5}=$ $k_{d 9}$ and $k_{d 6}=k_{d 10}$, the ODEs for $X_{t}$ and $Y_{t}$ are decoupled

$$
\begin{aligned}
\dot{X}_{t} & =\dot{X}_{a}+\dot{X}_{i}=-k_{d 5} \cdot X_{a}-k_{d 9} \cdot X_{i}+k_{p 9}=-k_{d 9} \cdot X_{t}+k_{p 9} \\
\dot{Y}_{t} & =\dot{Y}_{a}+\dot{Y}_{i}=-k_{d 6} \cdot Y_{a}-k_{d 10} \cdot Y_{i}+k_{p 10}=-k_{d 10} \cdot Y_{t}+k_{p 10}
\end{aligned}
$$

The system (1)-(4) is equivalent to the system (1)-(2), (7)-(8), i.e. we replace the ODEs for the inactive components by those of the total concentrations. Assuming the total concentrations to be in steady state, $X_{t}=k_{p 9} / k_{d 9}$ and $Y_{t}=k_{p 10} / k_{d 10}$. With these concentrations as initial conditions, $X_{t}$ and $Y_{t}$ are constant over time and the system can be reduced to two ODEs, (1) and (2). An equivalent system is obtained by considering relative concentrations, namely $X_{r}=X_{a} / X_{t}$ and $Y_{r}=Y_{a} / Y_{t}$. As an additional simplification, we assume $k_{d 9}=k_{d 10}=k_{d}$ and define $k_{1}=k_{c 1} \cdot Y_{t}$ and $k_{2}=k_{c 2} \cdot X_{t}^{n}$ to get

$$
\begin{gathered}
\dot{X}_{r}=k_{1} \cdot\left(1-X_{r}\right) \cdot Y_{r}-k_{d} \cdot X_{r} \\
\dot{Y}_{r}=k_{2} \cdot\left(1-Y_{r}\right) \cdot X_{r}^{n}-k_{d} \cdot Y_{r} .
\end{gathered}
$$

The nullclines of this system are

$$
\begin{array}{r}
X_{r}=\frac{k_{1} \cdot Y_{r}}{k_{d}+k_{1} \cdot Y_{r}}, \\
Y_{r}=\frac{k_{2} \cdot X_{r}^{n}}{k_{d}+k_{2} \cdot X_{r}^{n}} .
\end{array}
$$

Notice, these nullclines are equivalent to equations (5) and (6) under the above assumptions. Also notice, we started of with mass action kinetics and the steady state solutions have the same form as the well known Michaelis-Menten or Monod equation ((5) and (11)) and Hill equation ((6) and (12)) (Ferrell and Xiong, 2001).

In the following, we will proceed using the simplified system (9)-(10). The results obtained can be derived similarly for the more general case. The simplifications mainly affect the exact dynamics rather than the steady state behaviour. 


\subsection{Phase plane analysis}

Equation (11) is the nullcline to (9), and (12) is the nullcline to (10). In steady state, they also correspond to stimulus-response curves of two isolated subsystems, e.g. for (9) the steady state relation between the input (stimulus) $Y_{r}$ and the output (response) $X_{r}$ is given by (11). The steady states of the combined system (9)-(10) are characterized by the intersection of their nullclines (11)-(12).

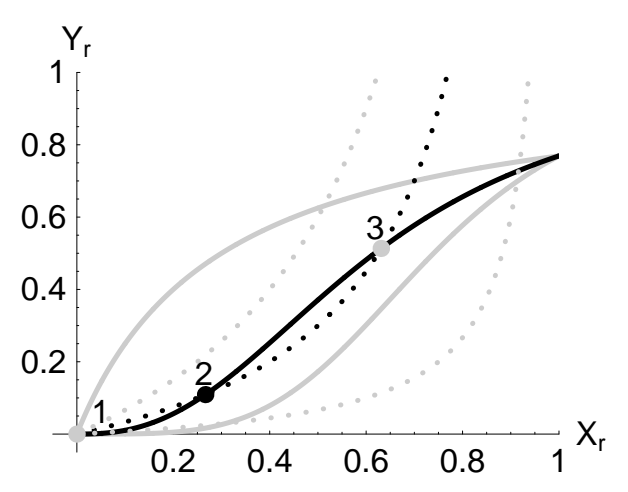

(a) $X_{r}$ and $Y_{r}$ nullclines.

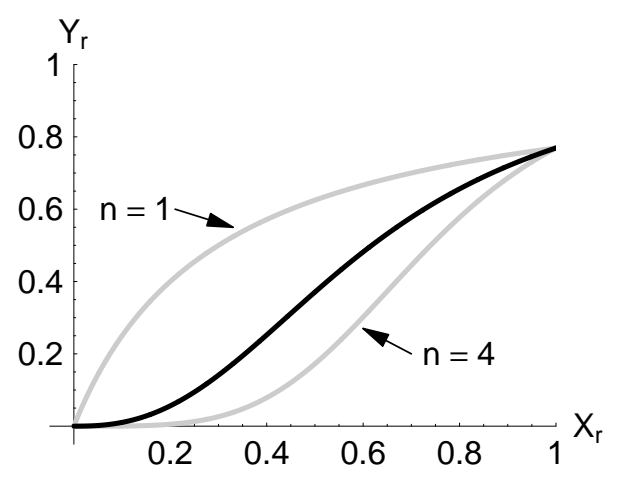

(c) $Y_{r}$ nullclines.

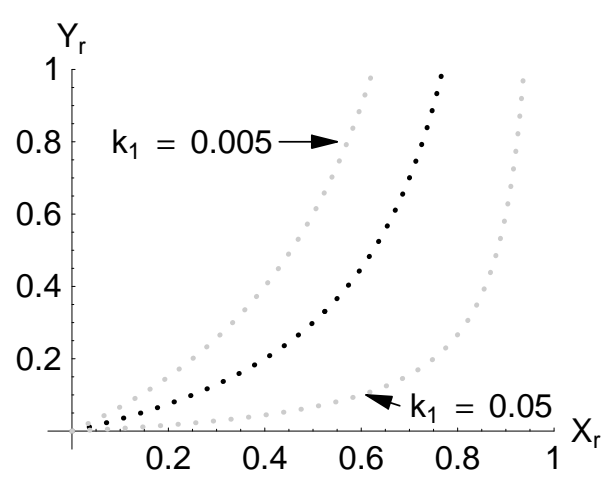

(b) $X_{r}$ nullclines.

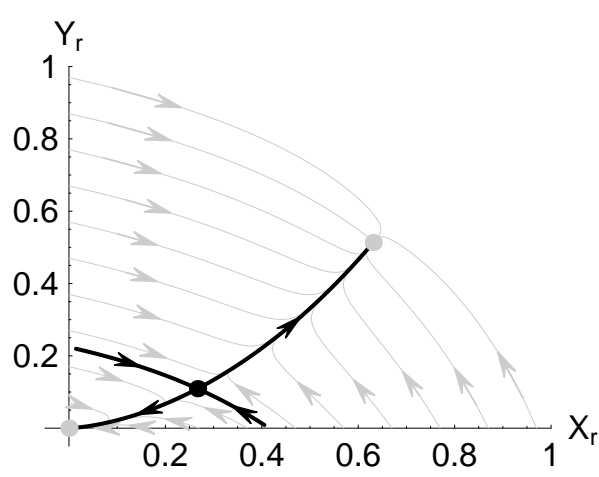

(d) Time trajectories.

Figure 2. Phase plane analysis. Fig. 2(a) is a superposition of Fig. 2(b) and 2(c) giving nullclines of (9) and (10), respectively. The numbered dots indicate steady states for one set of parameters. Fig. 2(d) illustrates trajectories of the system.

The $X_{r}$ nullcline is depicted in Fig. 2(b) for $k_{1}=0.01$ in black and for $k_{1}=$ 0.005 and 0.05 in grey. The $Y_{r}$ nullcline is depicted in Fig. 2(c) for $n=2.5$ in black and for $n=1$ and 4 in grey. Note that the $Y_{r}$ nullcline for $n=1$ and the black $X_{r}$ nullcline are symmetric with respect to the line $Y_{r}=X_{r}$ as $k_{1}=k_{2}$. Fig. 2(a) shows the superposition of the two nullcline plots. The intersections of the different nullclines provide the steady states as indicated by the three dots for the black lines. We number the steady states along the nullcline starting at the origin from 1 to 3 as indicated in the figure. 
The classical approach for determining the local stability properties of steady states is to calculate the eigenvalues of the linearised system or by finding a Lyapunov function in a neighbourhood of the steady state. Alternatively, simple graphical tests for phase plane plots exist for special system classes (Piccardi and Rinaldi, 2002).

The black nullclines correspond to $n=2.5$ and are typical for the case $n>1$. The grey dots in Fig. 2 correspond to stable steady states (1 and 3) and the black dot corresponds to an unstable steady state, which is a saddle (2). Thus the system is bistable. In most bistable systems, two stable steady states are separated by a third unstable steady state (Tyson and Othmer, 1978). Fig. 2(d) shows in black the unstable manifold of the saddle connecting the three steady states and the stable manifold separating the two areas of attraction. In addition, trajectories for different initial conditions are depicted in grey. For $n=1$ however, there are at most two steady states, thus bistability is not possible, irrespectively of the other parameters.

\section{Ways of generating bistability}

In Section 3, we derived the steady states and their stability properties for a reaction system of two mutually activating proteases. If the reaction system is modelled using mass action kinetics without cooperative steps, it is not able to produce a bistable behaviour. Key in generating bistable behaviour was the introduction of an ultrasensitive mechanism which was achieved through cooperativity. This leads to the deformation of a hyperbolic curve into a sigmoidal curve.

In the following, we will outline additional ways of generating a bistable behaviour in similarly simple reaction schemes. Two well described cases are zeroorder ultrasensitivity (Section 4.1) and inhibitor ultrasensitivity (Section 4.2) for which we directly show the simplified solution derived according to Section 3.2. Additionally, more complicated mechanisms have been investigated (Section 4.3).

\subsection{Zero-order ultrasensitivity}

Zero-order ultrasensitivity has so far mainly been studied in the context of kinase/phosphatase reaction schemes (Goldbeter and Koshland, 1981, 1984; Lisman, 1985). When the phosphatase kinetics are described by MichaelisMenten kinetics instead of mass action kinetics and the system is operating near saturation, this can lead to an ultrasensitive stimulus-response curve 
and thus to bistability in combination with positive feedback. Such a saturation behaviour is not possible for protease cleavage reactions due to their irreversibility. However, a closer comparison of the involved equations easily reveals that the scenario can be obtained when introducing a saturation in the degradation. To our knowledge there are no conclusive biological data available, but a saturation in the degradation can be envisioned as the degradation is usually carried out by the proteasome and either this machinery or upstream tagging enzymes could become saturated. We evaluate a saturation in the degradation of $Y_{r}$ assuming a constant concentration of the degrading enzyme $E_{d}$ and define $k_{m}=k_{m 6} \cdot E_{d} / Y_{t}$ and $K_{M}=K_{M 6} / Y_{t}$

$$
\dot{Y}_{r}=k_{2} \cdot\left(1-Y_{r}\right) \cdot X_{r}-\frac{k_{m} \cdot Y_{r}}{K_{M}+Y_{r}}
$$

where (9) as the second ODE remains unchanged. This yields the nullclines (11) and

$$
\begin{aligned}
Y_{r}= & \frac{k_{2} \cdot X_{r} \cdot\left(1-K_{M}\right)-k_{m}}{2 \cdot k_{2} \cdot X_{r}}+ \\
& \frac{\sqrt{\left(k_{2} \cdot X_{r} \cdot\left(1-K_{M}\right)-k_{m}\right)^{2}+4 \cdot K_{M} \cdot k_{2}^{2} \cdot X_{r}^{2}}}{2 \cdot k_{2} \cdot X_{r}}
\end{aligned}
$$

as well as a second negative and thus irrelevant solution. The $Y_{r}$ nullcline is depicted in Fig. 3(c) for $K_{M}=0.01$ in black and for $K_{M}=1$ and $10^{-4}$ in grey (computed with $X_{t}=Y_{t}=1$ ). Thus, although the biological interpretation strongly differs from previously described cases, saturation effects in combination with positive feedback can also produce a bistable behaviour in the here described setting. An introduction of a saturation in either of the cleavage reactions will on the other hand not be able to produce a bistable behaviour (data not shown).

\subsection{Inhibitor ultrasensitivity}

Inhibitor ultrasensitivity refers to the case where an inhibitor shapes the stimulus-response curve sigmoidal (Ferrell, 1996; Thron, 1994). This setup appears especially relevant for protease cascades as several specific inhibitors have been described for various proteases, e.g. IAPs inhibiting caspases (Danial and Korsmeyer, 2004) or Tissue Factor Pathway Inhibitor (TFPI) and antithrombin regulating important steps in the blood clotting cascade (Dahlbäck, 2000). We evaluate an inhibition where the inhibitor $I_{i}$ binds to and thereby inactivates the active protease $Y_{a}$. We define the relative amount of complex $I Y_{r}=I Y / Y_{t}, k_{f}=k_{f 3} \cdot Y_{t}$ and $k_{b}=k_{b 3}$. The ODEs for the complex and for 
$Y_{r}$ are given by

$$
\begin{aligned}
& I \dot{Y}_{r}=k_{f} \cdot\left(\frac{I_{t}}{Y_{t}}-I Y_{r}\right) \cdot Y_{r}-k_{b} \cdot I Y_{r}-k_{d} \cdot I Y_{r} \\
& \dot{Y}_{r}=k_{2} \cdot\left(1-Y_{r}-I Y_{r}\right) \cdot X_{r}-k_{d} \cdot Y_{r}-k_{f} \cdot\left(\frac{I_{t}}{Y_{t}}-I Y_{r}\right) \cdot Y_{r}+k_{b} \cdot I Y_{r} .
\end{aligned}
$$

Assuming the inhibitor and its complex to be in a quasi steady state, a substitution of $I Y_{r}$ into (16) yields

$$
\dot{Y}_{r}=k_{2} \cdot\left(1-Y_{r}\right) \cdot X_{r}-k_{d} \cdot Y_{r}-\frac{k_{f} \cdot I_{t} \cdot Y_{r} \cdot\left(k_{d}+k_{2} \cdot X_{r}\right)}{\left(k_{f} \cdot Y_{r}+k_{d}+k_{b}\right) \cdot Y_{t}}
$$

and in addition we keep (9) unchanged. This yields nullclines described by (11) and an equation for $Y_{r}$ provided in the supplement due to its length. As can be seen in Fig. 3(b) (computed with $X_{t}=Y_{t}=3 \cdot I_{t}=1$ ), this mechanism can also efficiently produce a bistable behaviour. The $Y_{r}$ nullcline is depicted for $k_{f}=1$ in black and for $k_{f}=0.1$ and 10 in grey. Compared to the cases where bistability is achieved through cooperative or zero-order ultrasensitivity the activation in the activated steady state is somewhat lower.

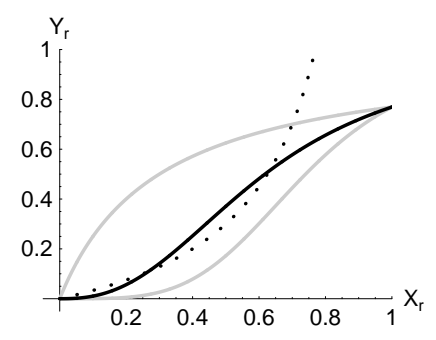

(a) Cooperative.

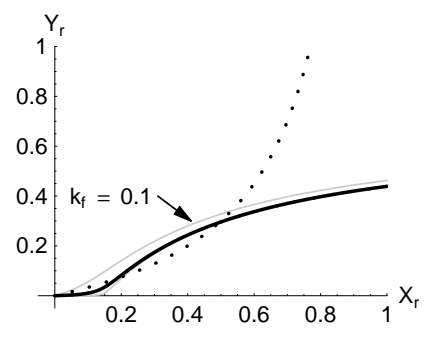

(b) Inhibitor.

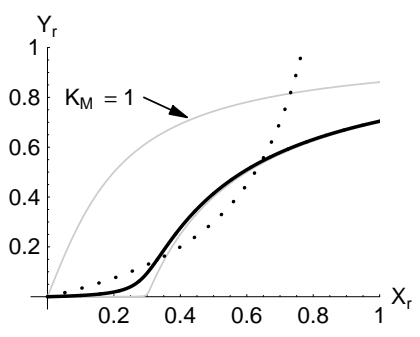

(c) Zero-order.

Figure 3. The three basic mechanisms to produce bistability: cooperative (3(a)), inhibitor (3(b)) and zero-order (3(c)) ultrasensitivity.

\subsection{Additional mechanisms and combinations}

Of course, there are theoretically many additional system configurations that can lead to bistability. Here we focused on positive feedback, however double negative feedback or more indirect or implicit forms of feedback can also yield a bistable behaviour (Ortega et al., 2002; Markevich et al., 2004; Angeli et al., 2004). Recently, this was also described for the mitochondrial pathway of apoptosis, where IAP proteins can inhibit both initiator and executioner caspases (Legewie et al., 2006). For monotone systems it was shown that any kind of feedback which in the sum is positive can give rise to a bistable behaviour (Angeli et al., 2004). As can be seen in Figs. 2 and 3, all these structural requirements are necessary but not sufficient for bistability. The different parameters have to be balanced in order to generate bistability. 
It is easy to envision that a combination of the different mechanisms can produce steeper sigmoidal curves (data not shown). Also, if the forward and the backward part of the reaction system contain a mechanism generating an ultrasensitive response this balance is more easily achieved, e.g. if not only $Y_{a}$ is inhibited by an inhibitor $I_{i}$ but also $X_{a}$ is inhibited by an inhibitor $I_{e}$ (data not shown, compare Eißing et al. (2004, 2005)).

\section{$5 \quad$ Bifurcation and hysteresis}

Instead of evaluating more complicated scenarios we rather would like to investigate more closely the dependence of the bistable behaviour on the parameters in different ways. We choose the model considering inhibitors as introduced in Section 4.2. The results are easily transferable to the other described scenarios.

Location and stability properties of steady states in dependence on parameters are usually evaluated in bifurcation diagrams. Such diagrams can be seen in Fig. 4. However, the qualitative picture can already be derived from Fig. 3(b). For example, we fix the solid black line and shift the dotted black line by changing $k_{1}$ (as indicated for two values by the dotted grey lines in Fig. 2(b)) corresponding to the positive feedback activation of $X$ by $Y$. For decreasing $k_{1}$, steady state 3 becomes smaller and the steady state 2 becomes larger until they finally meet and directly afterwards disappear (saddle-node bifurcation). If the feedback is too weak, a high activation state of the proteases cannot be sustained. On the other hand, for increasing $k_{1}$, steady state 2 becomes smaller until it finally reaches steady state 1 with which it exchanges its stability properties, before disappearing into the non-relevant negative orthant (transcritical bifurcation). If the feedback is too strong even the weakest activation causes the almost complete activation of the proteases. This behaviour is more quantitatively described in Fig. 4(a) where stable steady states are depicted as solid lines and unstable steady states as dashed lines. The physically irrelevant areas are hatched.

So far we considered that an external stimulus affects the initial conditions of the systems without explicitly considering the stimulus, e.g. $X_{a}$ is produced by some impulse stimulus. We now explicitly take the input into account and consider the stimulation of the system by a constant external stimulus. As can be seen in Fig. 4(b) the bifurcation diagram changes. The steady state 1 is now not always at zero but dependent on the external stimulus. The bifurcation diagram basically corresponds to a steady state response curve. When we slowly increase $U$ the system response also rises slowly as steady state 1 rises slowly. Once steady state 1 vanishes, the system jumps into steady state 3 and then again slowly rises further. If we now slowly lower $U$ we remain in steady state 3 and would only return to steady state 1 for negative $U$, i.e. 


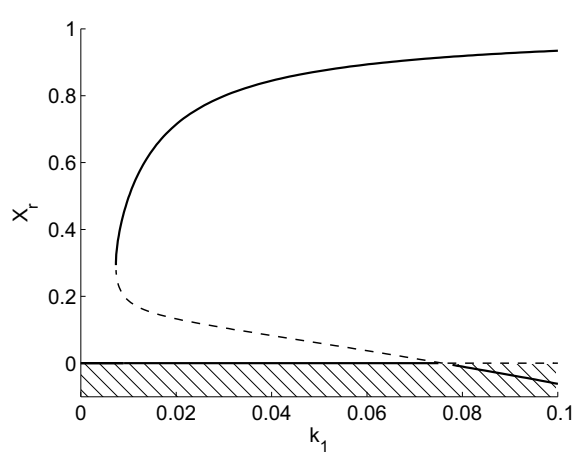

(a) $k_{1}$ bifurcation without input.

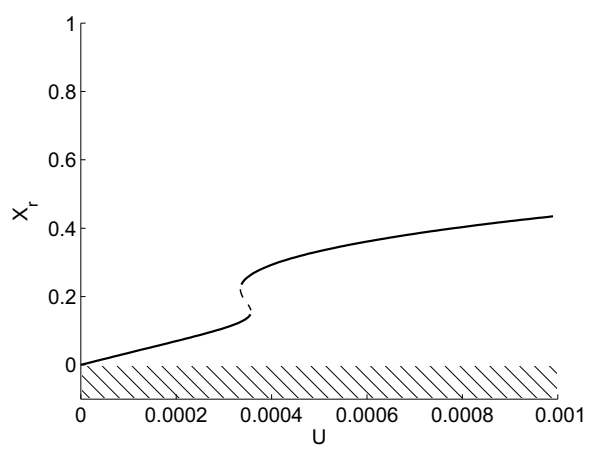

(c) Input bifurcation with $k_{1}=0.005$

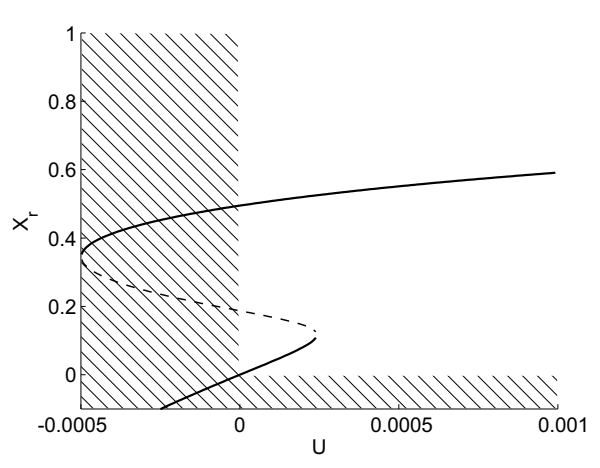

(b) Input bifurcation with $k_{1}=0.01$.

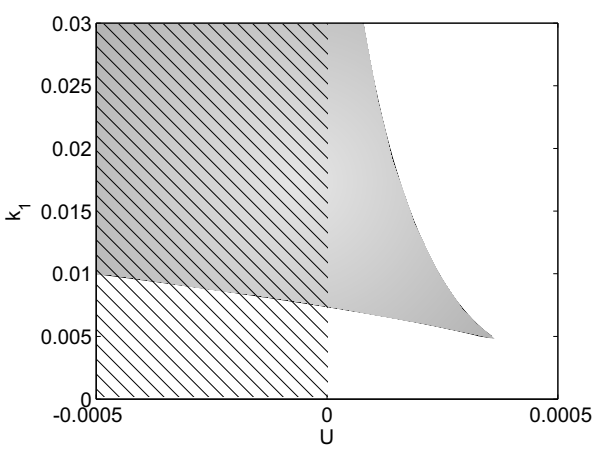

(d) Two parameter bifurcation.

Figure 4. Bifurcation analysis. Physically irrelevant areas are hatched. In 4(a) - 4(c) solid lines depict stable, dashed lines unstables steady states. In 4(d) the bistable region is filled in grey.

the hysteresis is large. If we assume $U \geq 0$, the system is basically trapped in steady state 3 - corresponding to a bistable behaviour of the system with $U \equiv 0$. The bifurcation diagram shows two saddle-node bifurcations. If we now choose a smaller $k_{1}$ as done for Fig. 4(c) we find the same behaviour but with a smaller hysteresis allowing the system to jump back to steady state 1 for small but positive values of $U$. The system is still bistable for some values of the constant input $U$ but is not irreversibly trapped for $U \geq 0$. Therefore, the system with $U \equiv 0$ is also not bistable (compare Fig. 2(a) and Fig. 3(b) for $\left.k_{1}=0.005\right)$.

Fig. 4(d) provides a two parameter bifurcation diagram. The two branches do not correspond to steady states any more, but indicate the bifurcation points as discussed before. The lower branch represents the left saddle-node bifurcation and the upper branch the right saddle-node bifurcation (compare to Figs. 4(b) and 4(c)). Between those branches, i.e. in the upper left part, the system is bistable, and it is monostable otherwise. As can be seen, the system is bistable for a large range of $k_{1}$ for $U \equiv 0$ but there is only a small range 
of $k_{1}$ allowing for a reversible switching in dependence on $U$, i.e. where both bifurcation events occur for $U \geq 0$.

\section{The influence of zymogenicity}

So far we have assumed that the inactive form, also called pro-form or zymogen, of the protease does not possess any catalytic activity. However, it is well know that most zymogens have a residual activity. This activity is called zymogenicity and is defined as

$$
\text { zymogenicity }=\frac{\text { activity of processed protease }}{\text { activity of zymogen }}
$$

with known values ranging from 2 to $10^{5}$ for different proteases (Stennicke and Salvesen, 1999). Next, we would like to evaluate the influence of this disturbance onto the system behaviour. To simplify the perturbation, we only consider that $X$ has a zymogen activity. The ODE for $Y_{r}$ for the cooperative model can be modified according to the definition of zymogenicity to give

$$
\dot{Y}_{r}=k_{2} \cdot\left(1-Y_{r}\right) \cdot X_{r}^{n}+\frac{1}{z} \cdot k_{2} \cdot\left(1-Y_{r}\right) \cdot\left(1-X_{r}\right)^{n}-k_{d} \cdot Y_{r} .
$$

Proceeding accordingly for the different ODEs for $Y_{r}((13)$ and (17)) and again performing a phase plane analysis shows that this disturbance can affect the bistable behaviour of the system.

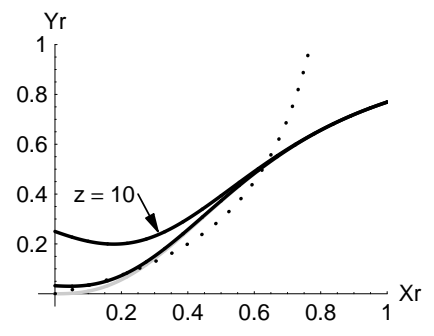

(a) Cooperative.

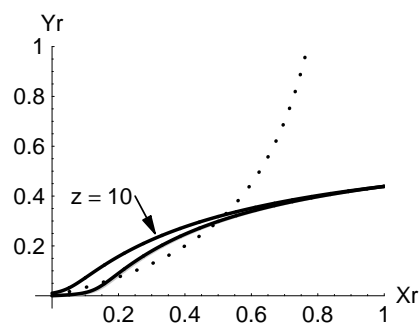

(b) Inhibitor.

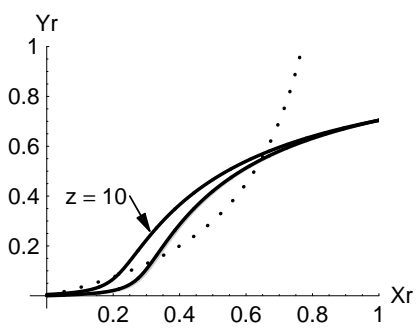

(c) Zero-order.

Figure 5 . The three basic mechanisms to generate bistability in the presence of zymogen activity: cooperative (Fig. 5(a)), inhibitor (Fig. 5(b)) and zero-order (Fig. 5(c)) ultrasensitivity. All plots show the $X_{r}$ nullcline in dashed black. The respective $Y_{r}$ nullclines are depicted for $z=\infty$ (no zymogen activity) as solid grey lines. These are buried by the solid black lines in Fig. 5(b) and 5(c) giving the $Y_{r}$ nullclines for $z=100$ and $z=10$.

Fig. 5 shows how the $Y_{r}$ nullclines are shifted when increasing zymogen activity (corresponding to decreasing zymogenicity) from $z=\infty$ (grey line), corresponding to the previously evaluated case with no zymogen activity, to $z=100$ and $z=10$ (black lines). For the inhibitor (Fig. 5(b)) and the zeroorder setup (Fig. 5(c)) the shift for $z=100$ is so minor that the nullclines 
bury the $z=\infty$ nullcline. Clearly, the cooperative case is severely affected (Fig. 5(a)).

\section{Discussion}

In this study we reviewed how a bistable behaviour can be generated in a small network of interacting proteins. Specifically, we chose two mutually activating proteases as an application example, which despite their widespread use in biological signalling networks have so far received little attention with respect to the generation of a bistable switch. The irreversibility of the involved reactions, as well as the need to consider protein turnover, render the example special compared to other well studied systems. Interestingly, the turnover can also be interpreted as a substitute for a back reaction, e.g. whereas in the classical case of zero-order ultrasensitivity the back reaction has to be close to saturation in order to generate bistability, the same has to hold for the degradation in our case. We have focused on two interacting proteases producing a bistable behaviour. It is easy to envision that even a single protease can generate a bistable switch similar to kinases (Ferrell and Xiong, 2001), when an autocatalytic mechanisms is introduced.

We employ phase plane and bifurcation analysis to illustrate the bistable behaviour from different viewpoints. Phase plane analysis generally relies on the reduction of the reaction system to second order. This reduction is not always possible. One interesting new concept routed in the theory of monotone systems is readily applicable to larger systems as has already been mentioned (Angeli et al., 2004; Angeli, 2006). However, the applicability of the concept is also restricted to special classes of systems. Both these graphical methods are mainly meant to get a qualitative picture, whereas bifurcation studies can be efficiently used to evaluate the parameter range for which the system actually does display a bistable behaviour. Bifurcation studies generally rely on continuation methods and numerical efficiency is the main limit. These studies can be performed in dependence on one or two parameters. Theoretically, the number of dimensions can be extended. The main restrictions are computational power and our visual conception. We can easily extend these analysis to three dimensions by solving the system of equations under the steady state condition for one variable, if the remaining equation has a special form (Eißing et al., 2004).

Another approach is to extract the bistability margins, i.e. basically the bifurcations points, for single parameter changes. This method can also yield measures for defining the robustness of the system (Ma and Iglesias, 2002). However, it relies on a reference parameter set. This reference parameter set has a strong impact on the outcome while it is usually not well defined. Re- 
cently, we have shown how a Monte Carlo based approach can provide comparable robustness measures without this limiting requirement (Eißing et al., 2005). However, the phase plane analysis can already outline principle robustness features of the here evaluated models. The robustness of the three models compared in Fig. 3 appears to mainly rely on the steepness of the ultrasensitive reaction component. In the models described here, this steepness is mainly depending on the number of molecules cooperating and their binding characteristics, the strength of the inhibitors binding or the saturation level as indicated by the Michaelis-Menten constant, respectively. However, the steepness is also affected by the chosen set of approximations (Goldbeter and Koshland, 1981; Blüthgen et al., 2006). In principle, none of the investigated mechanisms appears especially robust compared to another, although Bagci et al. (2006) report that the cooperative mechanism outperforms the inhibitor mechanism in a similar setup regarding the robustness of bistable behaviour with respect to parameter variations. When considering a class of perturbations so far mostly ignored, especially the cooperative setup appears very fragile (Fig. 5). We are therefore currently closer investigating the robustness aspects of the here described models.

From a biological point of view, all three mechanisms appear reasonable. Although there are several evidences, further experiments are needed to prove the existence of a protease switch within the cell (Eißing et al., 2004). Which mechanism is employed by nature and for what reason also awaits experimental elucidation. Clearly, many proteases are know to come along with specific inhibitors, e.g. caspases and IAPs. However, sometimes cooperative mechanisms appear to be involved in their activation, e.g. cytochrome c release and apoptosome assembly in the mitochondrial apoptotic pathway (Rehm et al., 2006). Likely, it will often turn out to be a combination of different mechanisms which ensure a robust and safe switch in vivo.

\section{Supplementary information}

Supplementary information is available at www.sysbio.de/projects/tnf/biosystems06/.

\section{Acknowledgement}

TE would like to thank Stefan Schuster for fruitful discussions and motivating this paper. Further, the authors would like to thank the Systems Biology group Stuttgart for fruitful discussions. This work was supported by the Center for Systems Biology Stuttgart and the Deutsche Forschungsgemeinschaft 
(DFG) grants BU 1405/1-1 and SCHE 349/8-1. EB acknowledges funding from Science Foundation Ireland (SFI) grant 03/RP1/I382.

\section{References}

Angeli, D., 2006. New analysis technique for multistability detection. Syst. Biol. 153 (2), 61-69.

Angeli, D., Ferrell, Jr., J. E., Sontag, E. D., 2004. Detection of multistability, bifurcations, and hysteresis in a large class of biological positive-feedback systems. Proc. Natl. Acad. Sci. U. S. A. 101 (7), 1822-1827.

Bagci, E. Z., Vodovotz, Y., Billiar, T. R., Ermentrout, G. B., Bahar, I., 2006. Bistability in apoptosis: Roles of Bax, Bcl-2 and mitochondrial permeability transition pores. Biophys. J. 90, 1546-1559.

Beltrami, E., Jesty, J., 1995. Mathematical analysis of activation thresholds in enzyme-catalyzed positive feedbacks: application to the feedbacks of blood coagulation. Proc. Natl. Acad. Sci. U. S. A. 92 (19), 8744-8748.

Bhalla, U. S., Ram, P. T., Iyengar, R., 2002. MAP kinase phosphatase as a locus of flexibility in a mitogen-activated protein kinase signaling network. Science 297 (5583), 1018-1023.

Blüthgen, N., Bruggeman, F. J., Legewie, S., Herzel, H., Westerhoff, H. V., Kholodenko, B. N., 2006. Effects of sequestration on signal transduction cascades. FEBS J. 273 (5), 895-906.

Cherry, J. L., Adler, F. R., 2000. How to make a biological switch. J. Theor. Biol. 203 (2), 117-133.

Dahlbäck, B., 2000. Blood coagulation. Lancet 355 (9215), 1627-1632.

Danial, N. N., Korsmeyer, S. J., 2004. Cell death: critical control points. Cell 116 (2), 205-219.

Dash, C., Kulkarni, A., Dunn, B., Rao, M., 2003. Aspartic peptidase inhibitors: implications in drug development. Crit. Rev. Biochem. Mol. Biol. 38 (2), 89-119.

Eißing, T., Allgöwer, F., Bullinger, E., 2005. Robustness properties of apoptosis models with respect to parameter variations and stochastic influences. Syst. Biol. 152 (4), 221-228.

Eißing, T., Conzelmann, H., Gilles, E. D., Allgöwer, F., Bullinger, E., Scheurich, P., 2004. Bistability analyses of a caspase activation model for receptor-induced apoptosis. J. Biol. Chem. 279 (35), 36892-36897.

Ferrell, Jr., J. E., 1996. Tripping the switch fantastic: how a protein kinase cascade can convert graded inputs into switch-like outputs. Trends Biochem. Sci. 21 (12), 460-466.

Ferrell, Jr., J. E., 1997. How responses get more switch-like as you move down a protein kinase cascade. Trends Biochem. Sci. 22 (8), 288-289.

Ferrell, Jr., J. E., Machleder, E. M., 1998. The biochemical basis of an all-ornone cell fate switch in Xenopus oocytes. Science 280 (5365), 895-898. 
Ferrell, Jr., J. E., Xiong, W., 2001. Bistability in cell signaling: How to make continuous processes discontinuous, and reversible processes irreversible. Chaos 11 (1), 227-236.

Goldbeter, A., Koshland, D. E., 1981. An amplified sensitivity arising from covalent modification in biological systems. Proc. Natl. Acad. Sci. U. S. A. 78 (11), 6840-6844.

Goldbeter, A., Koshland, D. E., 1984. Ultrasensitivity in biochemical systems controlled by covalent modification. Interplay between zero-order and multistep effects. J. Biol. Chem. 259 (23), 14441-14447.

Grabowskal, U., Chambers, T. J., Shiroo, M., 2005. Recent developments in cathepsin K inhibitor design. Curr Opin Drug Discov Devel 8 (5), 619-630.

Hengartner, M. O., 2000. The biochemistry of apoptosis. Nature 407 (6805), $770-6$.

Hodgkin, A. L., Huxley, A. F., 1952. A quantitative description of membrane current and its application to conduction and excitation in nerve. J. Physiol. 117 (4), 500-544.

Huang, C. Y., Ferrell, Jr., J. E., 1996. Ultrasensitivity in the mitogen-activated protein kinase cascade. Proc. Natl. Acad. Sci. U. S. A. 93 (19), 10078-83.

Koshland, D. E., 1998. The era of pathway quantification. Science 280 (5365), 852-853.

Legewie, S., Blüthgen, N., Herzel, H., 2006. Mathematical Modeling Identifies Inhibitors of Apoptosis as Mediators of Positive Feedback and Bistability. PLoS Comput. Biol. 2 (9), e120.

Leist, M., Jaattela, M., 2001. Four deaths and a funeral: from caspases to alternative mechanisms. Nat. Rev. Mol. Cell Biol. 2 (8), 589-98.

Lisman, J. E., 1985. A mechanism for memory storage insensitive to molecular turnover: a bistable autophosphorylating kinase. Proc. Natl. Acad. Sci. U. S. A. $82(9), 3055-7$.

Ma, L., Iglesias, P. A., 2002. Quantifying robustness of biochemical network models. BMC Bioinformatics 3, 38.

Mangan, S., Alon, U., 2003. Structure and function of the feed-forward loop network motif. Proc. Natl. Acad. Sci. U. S. A. 100 (21), 11980-5.

Markevich, N. I., Hoek, J. B., Kholodenko, B. N., 2004. Signaling switches and bistability arising from multisite phosphorylation in protein kinase cascades. J. Cell Biol. 164 (3), 353-359.

Ortega, F., Acerenza, L., Westerhoff, H. V., Mas, F., Cascante, M., 2002. Product dependence and bifunctionality compromise the ultrasensitivity of signal transduction cascades. Proc. Natl. Acad. Sci. U. S. A. 99 (3), 1170-5.

Overall, C. M., López-Otín, C., 2002. Strategies for MMP inhibition in cancer: innovations for the post-trial era. Nat. Rev. Cancer 2 (9), 657-672.

Piccardi, C., Rinaldi, S., 2002. Remarks on excitability, stability and sign of equilibria in cooperative systems. Syst. Cont. Lett. 46, 153-163.

Qu, Z., MacLellan, W. R., Weiss, J. N., 2003. Dynamics of the cell cycle: checkpoints, sizers, and timers. Biophys. J. 85 (6), 3600-3611.

Rehm, M., Huber, H. J., Dussmann, H., Prehn, J. H. M., 2006. Systems anal- 
ysis of effector caspase activation and its control by X-linked inhibitor of apoptosis protein. EMBO J. 25 (18), 4338-4349.

Shmulevich, I., Kauffman, S. A., Aldana, M., 2005. Eukaryotic cells are dynamically ordered or critical but not chaotic. Proc. Natl. Acad. Sci. U. S. A. 102 (38), 13439-13444.

Slee, E. A., Harte, M. T., Kluck, R. M., Wolf, B. B., Casiano, C. A., Newmeyer, D. D., Wang, H. G., Reed, J. C., Nicholson, D. W., Alnemri, E. S., Green, D. R., Martin, S. J., 1999. Ordering the cytochrome c-initiated caspase cascade: hierarchical activation of caspases-2, $-3,-6,-7,-8$, and -10 in a caspase-9-dependent manner. J. Cell Biol. 144 (2), 281-92.

Sloane, B. F., Yan, S., Podgorski, I., Linebaugh, B. E., Cher, M. L., Mai, J., Cavallo-Medved, D., Sameni, M., Dosescu, J., Moin, K., 2005. Cathepsin B and tumor proteolysis: contribution of the tumor microenvironment. Semin. Cancer Biol. 15 (2), 149-157.

Sohn, D., Schulze-Osthoff, K., Jänicke, R. U., 2005. Caspase-8 can be activated by interchain proteolysis without receptor-triggered dimerization during drug-induced apoptosis. J. Biol. Chem. 280 (7), 5267-5273.

Stelling, J., Sauer, U., Szallasi, Z., Dolye, 3rd, F. J., Doyle, J., 2004. Robustness of cellular functions. Cell 118 (6), 675-685.

Stennicke, H. R., Salvesen, G. S., 1999. Catalytic properties of the caspases. Cell Death Differ. 6 (11), 1054-1059.

Strogatz, S. H., 2001. Nonlinear dynamics and chaos: With applications to physics, biology, chemistry, and engineering. Perseus Books, Cambridge, MA.

Thron, C. D., 1994. Theoretical dynamics of the cyclin B-MPF system: a possible role for p13suc1. BioSystems 32 (2), 97-109.

Thron, C. D., 1997. Bistable biochemical switching and the control of the events of the cell cycle. Oncogene 15 (3), 317-25.

Thurmond, R. L., Sun, S., Karlsson, L., Edwards, J. P., 2005. Cathepsin S inhibitors as novel immunomodulators. Curr. Opin. Investig. Drugs 6 (5), $473-482$.

Tyson, J. J., Chen, K. C., Novak, B., 2003. Sniffers, buzzers, toggles and blinkers: dynamics of regulatory and signaling pathways in the cell. Curr. Opin. Cell Biol. 15 (2), 221-31.

Tyson, J. J., Othmer, H. G., 1978. Progress in Theoretical Biology. Vol. 5. Academic Press, New York, Ch. The dynamics of feedback control circuits in biochemical pathways, pp. 1-62.

Van de Craen, M., Declercq, W., Van den brande, I., Fiers, W., Vandenabeele, P., 1999. The proteolytic procaspase activation network: an in vitro analysis. Cell Death Differ. 6 (11), 1117-24.

Ventura, B. D., Lemerle, C., Michalodimitrakis, K., Serrano, L., 2006. From in vivo to in silico biology and back. Nature 443 (7111), 527-533.

Weiss, J. N., 1997. The Hill equation revisited: uses and misuses. FASEB J. 11 (11), 835-841.

Wolf, D. M., Arkin, A. P., 2003. Motifs, modules and games in bacteria. Curr. 
Opin. Microbiol. 6 (2), 125-134.

Xiong, W., Ferrell, Jr., J. E., 2003. A positive-feedback-based bistable 'memory module' that governs a cell fate decision. Nature 426 (6965), 460-5.

Yamashima, T., 2004. Ca2+-dependent proteases in ischemic neuronal death: a conserved 'calpain-cathepsin cascade' from nematodes to primates. Cell Calcium 36 (3-4), 285-293.

Yeger-Lotem, E., Sattath, S., Kashtan, N., Itzkovitz, S., Milo, R., Pinter, R. Y., Alon, U., Margalit, H., 2004. Network motifs in integrated cellular networks of transcription-regulation and protein-protein interaction. Proc. Natl. Acad. Sci. U. S. A. 101 (16), 5934-5939.

Zarnitsina, V. I., Ataullakhanov, F. I., Lobanov, A. I., Morozova, O. L., 2001. Dynamics of spatially nonuniform patterning in the model of blood coagulation. Chaos 11 (1), 57-70. 